\title{
Estradiol Synthesis and Metabolism and Risk of Ovarian Cancer in Older Women Taking Prescribed or Plant-derived Estrogen Supplementation
}

\section{Linda S M Gulliver*}

Faculty of Medicine, University of Otago, Dunedin, New Zealand

\begin{abstract}
Estradiol, the most potent of the biological estrogens, is implicated in the genesis of ovarian epithelial cancer, a heterogeneous cancer affecting mainly older women. The postmenopausal ovary traditionally has not been viewed as contributing significantly to estradiol synthesis, since this is thought to occur almost exclusively as the result of peripheral aromatization of adrenal androgens. Recent evidence supports a role for both normal and malignant ovarian tissue in de novo synthesis of estradiol using inactive biological precursors and available enzymatic pathways. The process is termed "intracrinology". The present paper reviews available evidence for the intracrinological synthesis of estradiol in ovarian surface epithelium. It further proposes how exogenous supplementation with synthetic hormone replacement may act to augment this process by increasing the risk of developing ovarian epithelial cancer in older women. Phytoestrogens are also examined for their role in regulating levels of estradiol metabolites with potent estrogenic and carcinogenic potential.
\end{abstract}

Keywords: Estradiol intracrinology synthesis/metabolism; Estrogen supplementation; Ovarian epithelial cancer; Older ovary; Ovarian surface epithelium

\begin{abstract}
Abbreviations: CYP: Cytochrome P450 Enzyme; HSD: Hydroxysteroid Dehydrogenase Enzyme; E1S: Estrone Sulfate; DHEA-S: Dehydroepiandosterone - sulfate; STS: Steroid Sulfatase; HGSC: High Grade Serous Carcinoma; RT-PCR: Reverse Transcription Polymerase Chain Reaction; OSE: Ovarian Surface Epithelium; EST: Estrogen Sulfotransferase; SHBG: Sex Hormone Binding Globulin; COMT: Catechol-O-Methyl Transferase; OVCAR-3: Ovarian Cancer 3 Cell Line; P13: Phosphatidyl-inositol 3; AKT: Protein kinase B; NFкB: Nuclear Factor Kappa B; ER: Estrogen Receptor
\end{abstract}

\section{Estradiol Synthesis in the Premenopausal Ovary}

All steroid hormones, of which estradiol is an example, are lipids that have cholesterol as their common precursory substrate. The synthesis of sex steroid hormones from cholesterol involves a series of sequential steps that result in the cleavage of side-chains, reorganization of olefinic bonds, and the addition of hydroxyl groups. For estradiol synthesis, this pathway is from cholesterol to pregnanes, then on to androstanes and arriving finally at the estranes [1]. Estradiol synthesis requires the activity of members of the Cytochrome P450 enzyme family (gene constructs CYPs 11,17 and 19), and a second family of enzymes, the hydroxysteroid dehydrogenases (HSD), which catalyze bi-directional reactions involved in both the biosynthesis and inactivation of steroid hormones. HSD exist in several different isoforms [2], and cooperate with steroid sulfotransferases, reductases and steroid sulfatases to regulate the level of bioactive hormone in all target tissues [1].

Estradiol synthesis in the premenopausal ovary requires the synergistic efforts of at least two cell types that synthesize their product when stimulated by independent upstream mechanisms. In theca, pregnenalone undergoes enzymatic conversion to androstenedione in a sequential fashion. Since thecal tissue lacks P450 aromatase (CYP 19) the final conversion to estradiol occurs in the adjacent granulosa cells and requires the actions of $17 \beta$ HSD1 in converting androstenedione to testosterone, after which aromatase completes the conversion through to estradiol $17 \beta[1]$. This pathway is subsequently termed "the aromatase pathway" for estradiol synthesis. In the luteal phase of the ovulatory cycle, mammalian theca and cells of the corpora lutea (theca lutein) act together with granulosa lutein cells of corpora lutea, to produce sizeable amounts of both estradiol and progesterone. Throughout the cycle, estrone-sulphate (E1S) and dehydroepiandosterone sulfate (DHEA-S - available in small amounts in the premenopausal ovary) may also undergo conversion to estrone and DHEA respectively via the sulfatases (STS). This is "the sulfatase pathway" for estradiol synthesis, since the actions of the sulfatases allows the final conversion through to estradiol to be completed by HSDs 3 and 17 and P450arom.

The localization and expression of the major enzymes required for ovarian steroidogenesis are shown in figure 1.

To summarize, the availability of cholesterol, the relative amounts and type of enzymes in each tissue or cell compartment, and the actions of FSH on granulosa cell [3] are the three variables upon which estradiol synthesis in the premenopausal mammalian ovary depends.

\section{Estradiol and the Postmenopausal Ovary: Potential for Oncogenesis}

Following menopause, peripheral estradiol levels in the blood are thought to be mainly due to contribution from the adrenals and peripheral aromatization of androgens to estrogen in adipose tissue and skin, where aromatase activity correlates with estradiol production. There is evidence however, that the postmenopausal ovary retains the ability to produce both androgens [4] and estrogen [5,6].

The degree to which ovarian surface epithelium (OSE) is capable of

*Corresponding author: Linda S M Gulliver, Faculty of Medicine, University of Otago, Dunedin, New Zealand, Tel: +64 3 470-4689; Fax: +64-3-479-5459; E-mail: linda.gulliver@otago.ac.nz

Received March 16, 2013; Accepted April 18, 2013; Published April 24, 2013

Citation: Gulliver LSM (2013) Estradiol Synthesis and Metabolism and Risk of Ovarian Cancer in Older Women Taking Prescribed or Plant-derived Estrogen Supplementation. J Steroids Hormon Sci S12: 003. doi:10.4172/2157-7536.S12003

Copyright: $\odot 2013$ Gulliver LSM. This is an open-access article distributed under the terms of the Creative Commons Attribution License, which permits unrestricted use, distribution, and reproduction in any medium, provided the original author and source are credited. 


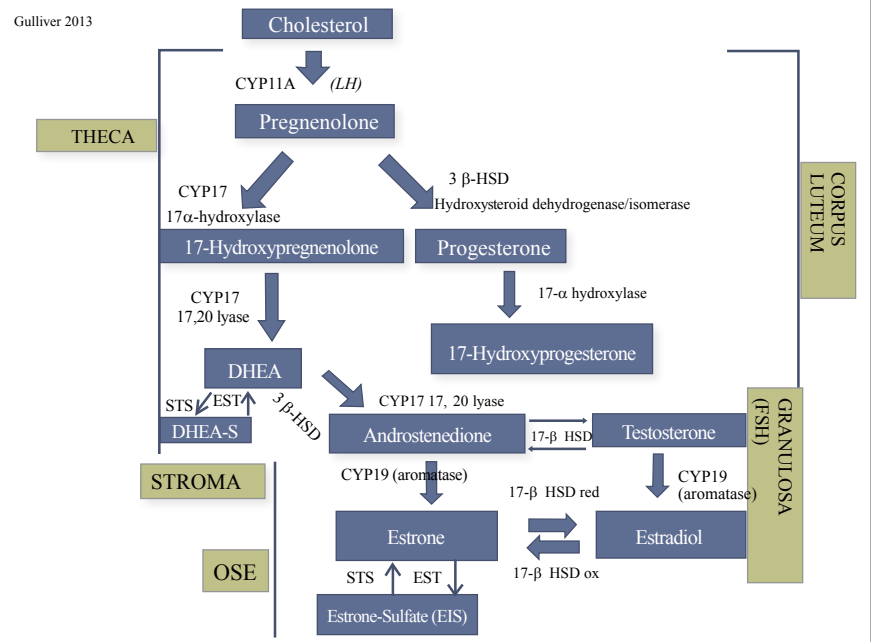

Figure 1: The ovary: Pathways of steroid hormone biosynthesis and the key enzymes involved.

steroidogenesis has only in recent years received attention, due largely to the OSE's unresolved role in ovarian epithelial cancer, a cancer of mainly older women. Although recent attention has turned to the adjacent fimbriated oviductal epithelium as being the tissue of origin for ovarian epithelial cancers (specifically high grade serous carcinomas (HGSC)) $[7,8]$, the most up-to-date evidence retains a definitive oncogenic role for mammalian OSE cells near the hilus of the ovary, at the point of OSE-mesothelial transition [9]. Since epidemiological evidence increasingly links estrogen to ovarian epithelial cancer [1018] and OSE has intracrinological capacity, the possibility of in situ estradiol-induced carcinogenesis in the postmenopausal ovary requires further investigation.

In vitro and in vivo evidence for aromatase activity in normal human OSE has been demonstrated using reverse transcription polymerase chain reaction (RT-PCR) [19,20] and immunohistochemistry $[20,21]$. Both benign and malignant ovarian epithelial tumors show immunohistochemical evidence of aromatase function in the cytoplasm of tumor cells [22], and aromatase is reported to be localized in stromal cells adjacent to ovarian carcinoma involving OSE [23]. In postmenopausal women, aromatase expression in normal OSE has been documented as six-fold higher than in stroma, and is also significantly greater in normal OSE when compared to malignant ovarian epithelial tumors [20]. This may indicate that when OSE undergoes malignant transformation, the aromatase pathway may not be the preferred pathway for the de novo synthesis of estradiol. Indeed the targeting of ovarian epithelial cancers with aromatase inhibitors has had mediocre success, perhaps not only due to the heterogeneity of ovarian cancers, but also because there is more than one player in localized estradiol synthesis. It has already been established that the sulfatase pathway is the dominant pathway for in situ estradiol synthesis in postmenopausal breast cancers, where it prevails over the aromatase pathway by some 50-200 fold [24].

Ovaries from older mice have been shown by aging-specific gene array to have increased not only mRNA levels of aromatase, but also progesterone receptor (PR) and estrogen sulfotransferase (EST) compared to younger mice. EST, an enzyme that catalyzes the conjugation of estrone to a sulphur group producing estrone sulfate (E1S, Figure 1), is the most abundant circulating estrogen conjugate and renders estrone inactive [25]. However, once the sulphur group is removed, estrone can bind to estrogen receptor but does so with low affinity. Interestingly, Zimon et al. [25] were not able to demonstrate increases in EST at protein level using Western Blot and immunofluorescence in older mouse ovary. Instead, they found levels of EST protein to be significantly reduced. EST mRNA expression has recently been reported to be markedly reduced in cultured primary ovarian epithelial cancer cells and all ovarian cancer cell lines when compared to normal OSE cells using quantitative RT-PCR [6].

Human OSE in cell culture additionally expresses steroid sulfatase (STS). STS works antagonistically to EST and converts E1S and dehydroepiandosterone-3 sulfate (DHEA-3-sulfate) to estrone and DHEA respectively (Figure 1). This raises the possibility that localized changes in the ratio of STS to EST in aging ovarian surface epithelium may boost levels of unconjugated estrone. Existing in vitro evidence using ovarian cancer cell lines, primary ovarian cancer cells and normal OSE, shows that activity of EST relative to STS is greater in normal OSE when compared to the cancerous state [6]. This finding may be important and invites more investigation into EST to STS ratios in OSE from postmenopausal women.

The significance of increased STS to EST ratio in OSE is that OSE also expresses mRNA for $17 \beta \mathrm{HSD}$ reductase required for the synthesis of estradiol from estrone [26]. In confluent culture, normal OSE from pre and postmenopausal women has been shown capable of de novo synthesis of both estrogen and progesterone using HSDs [27]. Thus, an increase in the STS to EST ratio may potentially boost local ovarian tissue levels of estradiol $17 \beta$, the far more potent biologically active estrogen that binds with strong affinity to its receptor. A current review targeting sulfatase activity for estrogen production in OSE supports this view [28] and underpins the emerging significance of the sulfatase pathway for generating potentially damaging levels of estradiol in OSE, conceivably predisposing it to oncogenesis.

It is possible that ovarian aging may have an effect on the stability of EST proteins. This may confer reduced ability to sulfonate estrone and estradiol obtained from peripheral blood via organic anion transporting polypeptides [28], which in turn may lead to increased levels of unconjugated estradiol in the ovary. Indeed in a recent study completed by our own lab, we found using radioimmunoassay, that some older mice had elevated levels of endogenous estradiol in ovarian tissue ( $>300 \mathrm{pg} / \mathrm{mL}$; normal range $66.2-117 \mathrm{pg} / \mathrm{mL}$ ). Furthermore, mice administered exogenous estradiol continued to show significantly elevated estradiol levels in ovarian tissue 2 weeks following cessation of estrogen treatment, indicating that unconjugated estradiol accumulates and is retained in ovarian tissue $[29,30]$.

These findings may go some way to providing an explanation as to why older women taking exogenous estradiol in the form of hormonal replacement therapy carry greater risk for the development of ovarian epithelial cancer. Friel et al. [31] reported that an oral estradiol dose regimen of between 1-2 $\mathrm{mg} /$ day can lead to a condition of estradiol overdose - as evidenced by urinary excretion of estrone 5-10 times the upper limit of the reference range for premenopausal women. Of note is that the present recommended estradiol dose in the U.S remains anywhere between $0.45 \mathrm{mg} /$ day $-2 \mathrm{mg} /$ day.

\section{Phytoestrogen Intake and Risk of Ovarian Epithelial Cancer}

While ample epidemiological evidence exists for hormone replacement therapies as having a role in the development of ovarian 
cancers, some evidence suggests that phytoestrogens taken in the diet may actually have the opposite effect. Phytoestrogens which include isoflavones (mostly soy-derived) and lignans (derived from grains, seeds, vegetables, fruits and berries) are established as having both estrogenic and anti-estrogenic properties [32,33]. Phytoestrogen consumption has been reported to either confer significantly reduced risk of ovarian cancer [34-36] show a non-significant inverse association with ovarian epithelial cancer [37], or show no association with overall ovarian epithelial cancer risk [38]. Interestingly in the latter prospective cohort study, fiber intake was associated with a decreased risk of borderline, but not invasive ovarian epithelial cancer [38]. Reuptake of estradiol metabolites found in bile is slowed by the presence of fiber in the intestine, lowering total estrogen. This mechanism for regulating estradiol may have little effect on established tumors capable of autonomous endocrine activity.

The differing results from the above studies may be a consequence of their differing methodologies (case-control based or prospective cohorts, dietary-derived only phytoestrogens versus dietary and supplement derived), different sample populations, age and menopausal status of the women. Moreover, ovarian cancers are highly heterogeneous and this has received little attention in the design and interpretation of these studies. Perhaps importantly, authors of the recent Swedish study [38] (where no association was seen between phytoestrogen intake and ovarian cancer risk) reported that overall bean/soy consumption in their cohort was low. Since other studies [3437] were able to show a dose-response correlation, whereby subjects making up the highest quintile of phytoestrogen consumption showed reduced risk for ovarian cancer compared to those in the lowest quintile, the normally low intake of phytoestrogens in the Swedish cohort may have influenced results from that study. Studies which report changes to estradiol metabolism that reduce risk for developing ovarian cancer when women ingest higher rather than lower quantities of phytoestrogens in the form of flaxseed (a lignan) and isoflavones $[39,40]$, lend further support for a protective role for phytoestrogens in the development of ovarian cancer.

There is an important emerging body of research investigating how ingested phytoestrogens act to control endogenous levels of estradiol by controlling the activity of estradiol's active metabolites. Circulating estradiol in human blood is controlled by regulatory mechanisms that act independently of the well-known endocrine feedback loops, including activin and inhibin. In humans, levels of Sex Hormone Binding Globulin (SHBG) act to bind and therefore control to some extent the actions of free unbound estradiol. Levels of SHBG increase with increasing levels of circulating estradiol and reflect the fact that more hormone is available for binding. The half-life of estradiol in the postmenopausal female is around 3 hours [41] with significant amounts of estradiol undergoing conversion to estriol and estrone followed by excretion of these well-known metabolites into the urine, bile and feces.

The formation of hydroxyl derivatives of estrogens (the catechol estrogens) during estradiol metabolism forms a potentially very important pathway for the control of endogenous estradiol levels. The principal hydroxylation products are 2-hydroxyestrone, 2-hydroxyestradiol, 4-hydroxyestrone, 4-hydroxyestradiol and 16-hydroxyestrone, 16-hydroxyestradiol [42]. Oxidation reactions allowing for the insertion of hydroxyl groups at positions 2-, 4-, or 16- are catalyzed by members of the Cytochrome P450 (CYP) family of enzymes. CYP1A1 catalyzes the 2-hydroxylation of estradiol and can be induced by dietary constituents [42]. CYP1B1 catalyzes both 16a- and 4-hydroxylation [42,43], and it has been reported that 16-hydroxyestradiol may be induced by pesticides and other xenobiotic carcinogens [42]. Estradiol catabolism continues when poly-hydroxyl entities undergo conjugation with sulfates and gluconarates, or are methylated via catechol-O-methyl transferase (COMT) before being excreted in the urine. However, 4-hydroxyestrogens are unstable, and can be oxidized by peroxidases to form highly reactive semi-quinone and quinone metabolites $[42,43]$.

Metabolite activity can be measured from samples of blood, tissue and urine. It is known that 2-hydroxyestrones are rapidly methylated in the blood by COMT and have anti-estrogenic (anti-proliferative) effects [43]. Although 4-hydroxyestrone and 4-hydroxyestradiol are found only in small amounts in the blood, they none-the-less have estrogenic action (hyperplasia, hypertrophy) and carcinogenic potential via the generation of free radicals [43-45]. The metabolite $16 a$-hydroxyesterone has been found to have potent estrogenic effects equivalent to, or stronger than, $17 \beta$-estradiol. These include DNA synthesis, persistent proliferation and anchorage-dependent growth $[42,46]$.

A diet that is high in soy isoflavones increases the $2 \alpha$-hydroxyestrone to $16 a$-hydroxyestrone ratio and lowers mid-cycle gonadotropin levels, leading to decreases in circulating estradiol, progesterone and SHBG in premenopausal women [47]. Decreases in 16a-hydroxyestrone, 4-hydroxyestrone and 4-hydroxyestradiol have also been shown in premenopausal women with high soy isoflavone intakes of $130 \mathrm{mg} /$ day compared to those with low intakes of 7-10 mg/day [40]. Moreover, the daily ingestion of large amounts $(10 \mathrm{~g})$ of ground flaxseed over a period of seven weeks in postmenopausal women has been reported to dramatically induce 2-hydroxylation of estrone and improve the ratio of 2/16a-hydroxyestrone, whereas only moderate effects were observed with reduced intakes of $5 \mathrm{~g}$ flaxseed per day [39]. Interestingly, supplementation with flaxseed lignans appears superior to soy in altering estrogen metabolism in postmenopausal women with respect to increasing $2 \alpha$ to $16 \alpha$ - hydroxylation. Flaxseed also moderately inhibits Cytochrome P450arom [48,49] and modulates the activity of the 17-HSD [50].

Some studies investigating estrogen metabolism and breast cancer have shown that estradiol metabolism favoring formation of 2-a hydroxylation over 16a-hydroxylation decreases the risk for developing breast cancer [51,52], although other studies show mixed results [53]. The differences in results may relate to the varying methodologies employed by researchers, but may also relate to menopausal status in the women studied. Recently, in an in vitro study using ovarian cancer cell line OVCAR-3 to examine the effects of the metabolites of estradiol on proliferation and apoptosis in comparison to estradiol itself, the $17 \beta$ proliferative and anti-apoptotic activity of the $16 \alpha$-hydroxylated estrone was shown to outstrip that of estradiol. Surprisingly $4 \alpha$-hydroxyestrone gave similar results to $17 \beta$ estradiol at physiologic concentrations [54], and may exert its effects through the PI3K/Akt signaling pathway to promote ovarian carcinogenesis. Importantly, the $2 \alpha$-hydroxyestrone metabolite was shown to have little activity. Although there is more research needed, these results indicate that the maintenance of pro-oncogenic to anti-oncogenic estradiol metabolites in the ovary may prove to be a very important factor in the genesis of ovarian epithelial cancer.

Finally, many phytoestrogens are known to bind both functional estrogen receptor subtypes (ER $\alpha$ and ER $\beta$ ), and are capable of inducing transcription of estrogen responsive target genes in a dose-dependent manner [55-58]. Whether phytoestrogen binding to ER produces 
Citation: Gulliver LSM (2013) Estradiol Synthesis and Metabolism and Risk of Ovarian Cancer in Older Women Taking Prescribed or Plant-derived Estrogen Supplementation. J Steroids Hormon Sci S12: 003. doi:10.4172/2157-7536.S12-003

the same or opposite effect to estradiol appears to depend on several factors: the type and amount of phytoestrogen [55-60], its relative binding affinity for the receptor subtype [56,57,61], the abundance from tissue to tissue of one ER subtype relative to the other [62], the presence of low-affinity (type II) nuclear binding sites [63], the ability of the phytoestrogen to utilize other non-genomic modalities (such as akt phosphorylation and $\mathrm{NFKB}$ ) to modulate estrogenic and carcinogenic effects $[64,65]$, and the presence of endogenous estrogen [66]. Activation of ERa by estradiol induces marked proliferation in normal and cancerous ovarian epithelial cells in vitro and in vivo [29,67-69], whereas activation of ER $\beta$ opposes the proliferative effects of ER $\alpha$ and has pro-apoptotic and anti-tumoral effects [69-71].

Phytoestrogens are known to bind ER with much lower affinity than estradiol [60,61], and preferentially bind ER $\beta$ [72]. Moreover, they induce the transcription of estrogen-responsive target genes to a much greater degree when bound to ER $\beta$, rather than when bound to ERa [66]. Phytoestrogens are also capable of inducing ER-mediated gene transcription to higher levels than estradiol itself [66]. Taken together, it may be proposed that in tissues such as ovary where ER $\beta$ is abundantly expressed, phytoestrogens may act to augment the anticarcinogenic effects of that receptor subtype. However, it should be noted that some phytoestrogens (e.g. genistein and resveratrol) have been known to act synergistically with estradiol in MCF-7 breast cancer cells [66,73], and can act as 'super agonists' that bind ERa as well as ER $\beta$. It is therefore important that further research defines the effects of different phytoestrogens on the ovary, and elucidates the cellular and molecular basis for their action.

\section{Conclusion}

At this time there appears to be a paucity of both in vitro and in vivo data for estradiol intracrinology and metabolite activity in the older female, and in the development of ovarian epithelial cancer. Since long-term exposure to estradiol is an established risk factor for ovarian cancer, this is an area of research that requires much more attention.

\section{References}

1. Strauss JFI, Lessey BA (2004) The structure, function, and evaluation of the female reproductive tract. In: Strauss JFI, Barbieri RL (eds.). Yen and Jaffe's Reproductive Endocrinology: physiology, pathophysiology and clinical management. (5thedn). Philadelphia.

2. Penning TM (1997) Molecular endocrinology of hydroxysteroid dehydrogenases. Endocr Rev 18: 281-305.

3. Carr BR (2004) The ovary and the normal menstrual cycle. Essential Reproductive Medicine. (1stedn), McGraw-Hill: 61-101.

4. Havelock JC, Rainey WE, Bradshaw KD, Carr BR (2006) The postmenopausal ovary displays a unique pattern of steroidogenic enzyme expression. Hum Reprod 21: 309-317.

5. Shifren JL, Schiff I (2000) The aging ovary. J Womens Health Gend Based Med 9 Suppl 1: S3-7.

6. Ren X, Harlow C, Fegan S, Mason I, Critchley H, et al. (2010) Expression and regulation of oestrogen sulfotransferase (EST) in human ovarian surface epithelium (OSE) and epithelial ovarian cancer. Manchester, UK.

7. Prat J (2012) New insights into ovarian cancer pathology. Ann Oncol 23: x111117

8. Kurman RJ, Shih leM (2011) Molecular pathogenesis and extraovarian origin of epithelial ovarian cancer--shifting the paradigm. Hum Pathol 42: 918-931.

9. Flesken-Nikitin A, Hwang $\mathrm{Cl}$, Cheng CY, Michurina TV, Enikolopov G, et al (2013) Ovarian surface epithelium at the junction area contains a cancer-prone stem cell niche. Nature 495: 241-245.

10. Hoover R, Gray LA Sr, Fraumeni JF Jr (1977) Stilboestrol (diethylstilbestrol) and the risk of ovarian cancer. Lancet 2: 533-534.
11. Rodriguez C, Patel AV, Calle EE, Jacob EJ, Thun MJ (2001) Estrogen replacement therapy and ovarian cancer mortality in a large prospective study of US women. JAMA 285: 1460-1465.

12. Lacey JV Jr, Mink PJ, Lubin JH, Sherman ME, Troisi R, et al. (2002) Menopausal hormone replacement therapy and risk of ovarian cancer. JAMA 288: 334-341.

13. Riman T, Dickman PW, Nilsson S, Correia N, Nordlinder H, et al. (2002) Hormone replacement therapy and the risk of invasive epithelial ovarian cancer in Swedish women. J Natl Cancer Inst 94: 497-504.

14. Folsom AR, Anderson JP, Ross JA (2004) Estrogen replacement therapy and ovarian cancer. Epidemiology 15: 100-104.

15. Mills PK, Riordan DG, Cress RD (2004) Epithelial ovarian cancer risk by invasiveness and cell type in the Central Valley of California. Gynecol Oncol 95: $215-225$

16. Moorman PG, Schildkraut JM, Calingaert B, Halabi S, Berchuck A (2005) Menopausal hormones and risk of ovarian cancer. Am J Obstet Gynecol 193: 76-82

17. Lacey JV Jr, Brinton LA, Leitzmann MF, Mouw T, Hollenbeck A, et al. (2006) Menopausal hormone therapy and ovarian cancer risk in the National Institutes of Health-AARP Diet and Health Study Cohort. J Natl Cancer Inst 98: $1397-$ 1405

18. Pearce CL, Chung K, Pike MC, Wu AH (2009) Increased ovarian cancer risk associated with menopausal estrogen therapy is reduced by adding a progestin. Cancer 115: 531-539.

19. Imai A, Ohno T, Takahashi K, Furui T, Tamaya T (1994) Lack of evidence for aromatase expression in human ovarian epithelial carcinoma. Ann Clin Biochem 31: 65-71.

20. Cunat S, Rabenoelina F, Daurès JP, Katsaros D, Sasano H, et al. (2005) Aromatase expression in ovarian epithelial cancers. J Steroid Biochem Mol Biol 93: 15-24.

21. Okubo T, Mok SC, Chen S (2000) Regulation of aromatase expression in human ovarian surface epithelial cells. J Clin Endocrinol Metab 85: 4889-4899.

22. Kitawaki J, Noguchi T, Yamamoto T, Yokota K, Maeda K, et al. (1996) Immunohistochemical localisation of aromatase and its correlation with progesterone receptors in ovarian epithelial tumours. Anticancer Res 16: 91 97.

23. Kaga K, Sasano H, Harada N, Ozaki M, Sato S, et al. (1996) Aromatase in human common epithelial ovarian neoplasms. Am J Pathol 149: 45-51.

24. Pasqualini JR, Chetrite G, Blacker C, Feinstein MC, Delalonde L, et al. (1996) Concentrations of estrone, estradiol, and estrone sulfate and evaluation of sulfatase and aromatase activities in pre- and postmenopausal breast cancer patients. J Clin Endocrinol Metab 81: 1460-1464.

25. Zimon A, Erat A, Von Wald T, Bissell B, Koulova A, et al. (2006) Genes invoked in the ovarian transition to menopause. Nucleic Acids Res 34: 3279-3287.

26. Rae MT, Hillier SG (2005) Steroid signalling in the ovarian surface epithelium Trends Endocrinol Metab 16: 327-333.

27. Ivarsson K, Sundfeldt K, Brännström M, Janson PO (2001) Production of steroids by human ovarian surface epithelial cells in culture: possible role of progesterone as growth inhibitor. Gynecol Oncol 82: 116-121.

28. Secky L, Svoboda M, Klameth L, Bajna E, Hamilton G, et al. (2013) The sulfatase pathway for estrogen formation: targets for the treatment and diagnosis of hormone-associated tumors. J Drug Deliv 2013: 957605.

29. Gulliver LS, Hurst PR (2011) Novel approaches to quantify estradiol-induced loss of ERß1 protein in older mouse ovarian surface epithelium: new tools to assess the role of ER protein subtypes in predisposing to ovarian epithelial cancer? Horm Cancer 2: 204-213.

30. Gulliver LS, Hurst PR (2012) Repeat estradiol exposure differentially regulates protein expression patterns for estrogen receptor and E-cadherin in older mouse ovarian surface epithelium: implications for replacement and adjuvant hormone therapies? Steroids 77: 674-685.

31. Friel PN, Hinchcliffe C, Wright JV (2005) Hormone replacement with estradiol: conventional oral doses result in excessive exposure to estrone. Altern Med Rev 10: 36-41

32. Mazur W, Adlercreutz H (2000) Overview of naturally occurring endocrineactive substances in the human diet in relation to human health. Nutrition 16 : 654-658. 
Citation: Gulliver LSM (2013) Estradiol Synthesis and Metabolism and Risk of Ovarian Cancer in Older Women Taking Prescribed or Plant-derived Estrogen Supplementation. J Steroids Hormon Sci S12: 003. doi:10.4172/2157-7536.S12-003

Page 5 of 5

33. Mazur W (1998) Phytoestrogen content in foods. Baillieres Clin Endocrinol Metab 12: 729-742.

34. McCann SE, Freudenheim JL, Marshall JR, Graham S (2003) Risk of human ovarian cancer is related to dietary intake of selected nutrients, phytochemicals and food groups. J Nutr 133: 1937-1942.

35. Chang ET, Lee VS, Canchola AJ, Clarke CA, Purdie DM, et al. (2007) Diet and risk of ovarian cancer in the California Teachers Study cohort. Am J Epidemiol 165: 802-813.

36. Rossi M, Negri E, Lagiou P, Talamini R, Dal Maso L, et al. (2008) Flavonoids and ovarian cancer risk: A case-control study in Italy. Int J Cancer 123: 895898.

37. Bandera EV, King M, Chandran U, Paddock LE, Rodriguez-Rodriguez L, et al (2011) Phytoestrogen consumption from foods and supplements and epithelial ovarian cancer risk: a population-based case control study. BMC Womens Health 11: 40.

38. Hedelin M, Löf M, Andersson TM, Adlercreutz H, Weiderpass E (2011) Dietary phytoestrogens and the risk of ovarian cancer in the women's lifestyle and health cohort study. Cancer Epidemiol Biomarkers Prev 20: 308-317.

39. Haggans CJ, Hutchins AM, Olson BA, Thomas W, Martini MC, et al. (1999) Effect of flaxseed consumption on urinary estrogen metabolites in postmenopausal women. Nutr Cancer 33: 188-195.

40. Xu X, Duncan AM, Merz BE, Kurzer MS (1998) Effects of soy isoflavones on estrogen and phytoestrogen metabolism in premenopausal women. Cancer Epidemiol Biomarkers Prev 7: 1101-1108.

41. Ginsburg ES, Gao X, Shea BF, Barbieri RL (1998) Half-life of estradiol in postmenopausal women. Gynecol Obstet Invest 45: 45-48.

42. Lord RS, Bongiovanni B, Bralley JA (2002) Estrogen metabolism and the dietcancer connection: rationale for assessing the ratio of urinary hydroxylated estrogen metabolites. Altern Med Rev 7: 112-129.

43. Tsuchiya Y, Nakajima M, Yokoi T (2005) Cytochrome P450-mediated metabolism of estrogens and its regulation in human. Cancer Lett 227: 115124.

44. Lippert C, Seeger H, Mueck AO, Lippert TH (2000) The effects of A-ring and $D$-ring metabolites of estradiol on the proliferation of vascular endothelial cells. Life Sci 67: 1653-1658.

45. Seeger H, Deuringer FU, Wallwiener D, Mueck AO (2004) Breast cancer risk during HRT: influence of estradiol metabolites on breast cancer and endothelial cell proliferation. Maturitas 49: 235-240.

46. Fishman J, Martucci C (1980) Biological properties of 16 alpha-hydroxyestrone: implications in estrogen physiology and pathophysiology. J Clin Endocrinol Metab 51: 611-615

47. Kurzer MS (2002) Hormonal effects of soy in premenopausal women and men J Nutr 132: 570S-573S.

48. Adlercreutz H, Bannwart C, Wähälä K, Mäkelä T, Brunow G, et al. (1993) Inhibition of human aromatase by mammalian lignans and isoflavonoid phytoestrogens. J Steroid Biochem Mol Biol 44: 147-153.

49. Wang C, Mäkelä T, Hase T, Adlercreutz H, Kurzer MS (1994) Lignans and flavonoids inhibit aromatase enzyme in human preadipocytes. J Steroid Biochem Mol Biol 50: 205-212.

50. Evans BA, Griffiths K, Morton MS (1995) Inhibition of 5 alpha-reductase in genital skin fibroblasts and prostate tissue by dietary lignans and isoflavonoids. J Endocrinol 147: 295-302.

51. Muti $\mathrm{P}$, Bradlow HL, Micheli A, Krogh V, Freudenheim JL, et al. (2000) Estrogen metabolism and risk of breast cancer: a prospective study of the 2:16alphahydroxyestrone ratio in premenopausal and postmenopausal women. Epidemiology 11: 635-640.

52. Zheng W, Dunning L, Jin F, Holtzman J (1998) Correspondence re: G. C. Kabat et al., Urinary estrogen metabolites and breast cancer: a case-control study. Cancer Epidemiol., Biomark. Prev., 6: 505-509, 1997. Cancer Epidemiol Biomarkers Prev 7: 85-86.

This article was originally published in a special issue, Steroid Hormone Metabolism handled by Editor. Dr. Carin Wittnich, University of Toronto, Canada
53. Seeger H, Mueck AO (2010) Estradiol metabolites and their possible role in gynaecological cancer. J Repro Med Endocr 7: 62-66

54. Seeger H, Wallwiener D, Kraemer E, Mueck AO (2006) Comparison of possible carcinogenic estradiol metabolites: effects on proliferation, apoptosis and metastasis of human breast cancer cells. Maturitas 54: 72-77.

55. Bowers JL, Tyulmenkov VV, Jernigan SC, Klinge CM (2000) Resveratro acts as a mixed agonist/antagonist for estrogen receptors alpha and beta. Endocrinology 141: 3657-3667.

56. Kuiper GG, Carlsson B, Grandien K, Enmark E, Häggblad J, et al. (1997) Comparison of the ligand binding specificity and transcript tissue distribution of estrogen receptors alpha and beta. Endocrinology 138: 863-870.

57. Kuiper GG, Lemmen JG, Carlsson B, Corton JC, Safe SH, et al. (1998) Interaction of estrogenic chemicals and phytoestrogens with estrogen receptor beta. Endocrinology 139: 4252-4263.

58. Maggiolini M, Bonofiglio D, Marsico S, Panno ML, Cenni B, et al. (2001) Estrogen receptor alpha mediates the proliferative but not the cytotoxic dosedependent effects of two major phytoestrogens on human breast cancer cells. Mol Pharmacol 60: 595-602.

59. Lehmann L, Esch HL, Wagner J, Rohnstock L, Metzler M (2005) Estrogenic and genotoxic potential of equol and two hydroxylated metabolites of Daidzein in cultured human Ishikawa cells. Toxicol Lett 158: 72-86.

60. van der Woude H, Ter Veld MG, Jacobs N, van der Saag PT, Murk AJ, et al. (2005) The stimulation of cell proliferation by quercetin is mediated by the estrogen receptor. Mol Nutr Food Res 49: 763-771.

61. McCarty MF (2006) Isoflavones made simple - genistein's agonist activity for the beta-type estrogen receptor mediates their health benefits. Med Hypotheses 66: 1093-1114.

62. McDonnell DP (2004) The molecular determinants of estrogen receptor pharmacology. Maturitas 48 Suppl 1: S7-12

63. Markaverich BM, Shoulars K, Brown MA (2001) Purification and characterization of nuclear type II [(3)H]estradiol binding sites from the rat uterus: covalent labeling with $[(3) \mathrm{H}]$ luteolin. Steroids 66: 707-719.

64. Brownson DM, Azios NG, Fuqua BK, Dharmawardhane SF, Mabry TJ (2002) Flavonoid effects relevant to cancer. J Nutr 132: 3482S-3489S.

65. Banerjee S, Bueso-Ramos C, Aggarwal BB (2002) Suppression of 7,12-dimethylbenz(a)anthracene-induced mammary carcinogenesis in rats by resveratrol: role of nuclear factor-kappaB, cyclooxygenase 2, and matrix metalloprotease 9. Cancer Res 62: 4945-4954.

66. Harris DM, Besselink E, Henning SM, Go VL, Heber D (2005) Phytoestrogens induce differential estrogen receptor alpha- or Beta-mediated responses in transfected breast cancer cells. Exp Biol Med (Maywood) 230: 558-568.

67. Bai W, Oliveros-Saunders B, Wang Q, Acevedo-Duncan ME, Nicosia SV (2000) Estrogen stimulation of ovarian surface epithelial cell proliferation. In Vitro Cell Dev Biol Anim 36: 657-666.

68. O'Donnell AJ, Macleod KG, Burns DJ, Smyth JF, Langdon SP (2005) Estrogen receptor-alpha mediates gene expression changes and growth response in ovarian cancer cells exposed to estrogen. Endocr Relat Cancer 12: 851-866.

69. Bossard C, Busson M, Vindrieux D, Gaudin F, Machelon V, et al. (2012 Potential role of estrogen receptor beta as a tumor suppressor of epithelial ovarian cancer. PLoS One 7: e44787.

70. Lazennec G, Bresson D, Lucas A, Chauveau C, Vignon F (2001) ER beta inhibits proliferation and invasion of breast cancer cells. Endocrinology 142 4120-4130.

71. Lazennec G (2006) Estrogen receptor beta, a possible tumor suppressor involved in ovarian carcinogenesis. Cancer Lett 231: 151-157.

72. Cassidy A (2003) Potential risks and benefits of phytoestrogen-rich diets. Int Vitam Nutr Res 73: 120-126.

73. Gehm BD, McAndrews JM, Chien PY, Jameson JL (1997) Resveratrol, a polyphenolic compound found in grapes and wine, is an agonist for the estrogen receptor. Proc Natl Acad Sci USA 94: 14138-14143. 